\title{
Two polyketide-synthase-associated acyltransferases are required for sulfolipid biosynthesis in Mycobacterium tuberculosis
}

\begin{abstract}
Correspondence
William R. Jacobs, Jr

jacobsw@hhmi.org
\end{abstract}

Received 3 October 2006

Revised 18 October 2006

Accepted 30 October 2006

\author{
Kiranmai Bhatt, ${ }^{1}$ Sudagar S. Gurcha, ${ }^{2}$ Apoorva Bhatt, ${ }^{1}$ Gurdyal S. Besra ${ }^{2}$ \\ and William R. Jacobs, Jr ${ }^{1}$
}

\author{
${ }^{1}$ Howard Hughes Medical Institute, Department of Microbiology and Immunology, Albert \\ Einstein College of Medicine, 1300 Morris Park Avenue, Bronx, NY 10461, USA
}

${ }^{2}$ School of Biosciences, University of Birmingham, Edgbaston, Birmingham B15 2TT, UK

\begin{abstract}
The methyl-branched fatty acyl components of sulfolipid-I (SL-I), a major glycolipid of the human pathogen Mycobacterium tuberculosis, are synthesized by the polyketide synthase Pks2. Rv3824c (papA1), located downstream of $p k s 2$, encodes a protein that belongs to a subfamily of acyltransferases associated with mycobacterial polyketide synthases [polyketide synthaseassociated proteins (PAPs)]. The presence of a conserved acyltransferase motif $\left(\mathrm{HX}_{3} \mathrm{DX}{ }_{14} \mathrm{Y}\right)$ suggested a role for PapA1 in acylation of sulfated trehalose to form SL-I. Targeted deletion of the H37Rv papA1 resulted in loss of SL-I, demonstrating its role in mycobacterial sulfolipid biosynthesis. Furthermore, SL-I synthesis was restored in the mutant strain following complementation with papA1, but not with mutant alleles of papA1 containing alterations of key residues in the acyltransferase motif, confirming that PapA1 was an acyltransferase. While other $M$. tuberculosis pks clusters are associated with a single PAP-encoding gene, it was demonstrated that another open reading frame, Rv3820c (papA2), located $5.8 \mathrm{~kb}$ downstream of papA1 is also an acyltransferase gene involved in SL-I biosynthesis: deletion of papA2 abolished SL-I production. The absence of any partially acylated intermediates in either null mutant indicated that both PapA1 and PapA2 were required for all acylation steps of SL-I assembly.
\end{abstract}

\section{INTRODUCTION}

Mycobacterium tuberculosis, the causative agent of tuberculosis, has a distinct, lipid-rich cell wall that plays an important role in virulence (Brennan \& Nikaido, 1995; Puzo, 1990). Sulfolipids are one of the major M. tuberculosis cell wall lipids, of which sulfolipid-I (SL-I) is the most abundant (Goren, 1970a, b; Goren et al., 1976; Middlebrook et al., 1959). The presence of sulfolipids in virulent strains led to the speculation that this class of polar lipids plays an important role in pathogenesis. However, there have been mixed reports about the role of sulfolipids in virulence, with various modulatory effects being observed with purified sulfolipids (Okamoto et al., 2006; Pabst et al., 1988; Zhang et al., 1988), but no attenuation observed with mutant strains in mouse and guinea pig models of infection (Rousseau et al., 2003). SL-I consists of a tetra-acylated trehalose sulfate (Fig. 1): of the four acyl groups that are esterified to trehalose, one is palmitate while the remaining three are complex methyl-branched fatty acids, termed phthioceranic and hydroxyphthioceranic acids (Goren et al.,

Abbreviations: FAS-I, fatty acid synthase-I; PAP, polyketide synthaseassociated protein; PDIM, phthiocerol dimycocerosate; SL-I, sulfolipid-I.
1971). The sulfation of trehalose is presumed to be the first step in SL-I assembly and is catalysed by the sulfotransferase Stf0 (Mougous et al., 2004). The palmitate is fatty acid synthase-I (FAS-I)-derived, the methyl-branched moieties, phthioceranic and hydroxyphthioceranic acids, are synthesized by a polyketide synthase, Pks2 (Sirakova et al., 2001). Present in the same cluster as pks2 is mmpL8, a gene encoding a transmembrane protein involved in transport (Converse et al., 2003; Domenech et al., 2004). Though the genes involved in the biosynthesis of individual components of SL-I are well studied, not much is known about the assembly of SL-I, vis-à-vis the components and sequence of events involved in acylation of trehalose-2-sulfate to SL-I. Located immediately downstream of $p k s 2$ is $R v 3824 c$ (papA1; Fig. 2a), which encodes a protein that belongs to a subfamily of acyltransferases associated with mycobacterial polyketide synthases [polyketide synthase associated proteins (PAPs)]. The presence of a characteristic $\mathrm{HX}_{3} \mathrm{DX}_{14} \mathrm{Y}$ acyltransferase motif suggested a role for PapA1 in the acylation steps of SL-I assembly. Indeed, PapA5, another PAP, has been shown to be an acyltransferase involved in the esterification of phthiocerol with methylbranched mycocerosic fatty acids to form the virulence lipid phthiocerol dimycocerosate (PDIM) (Onwueme et al., 2004; 


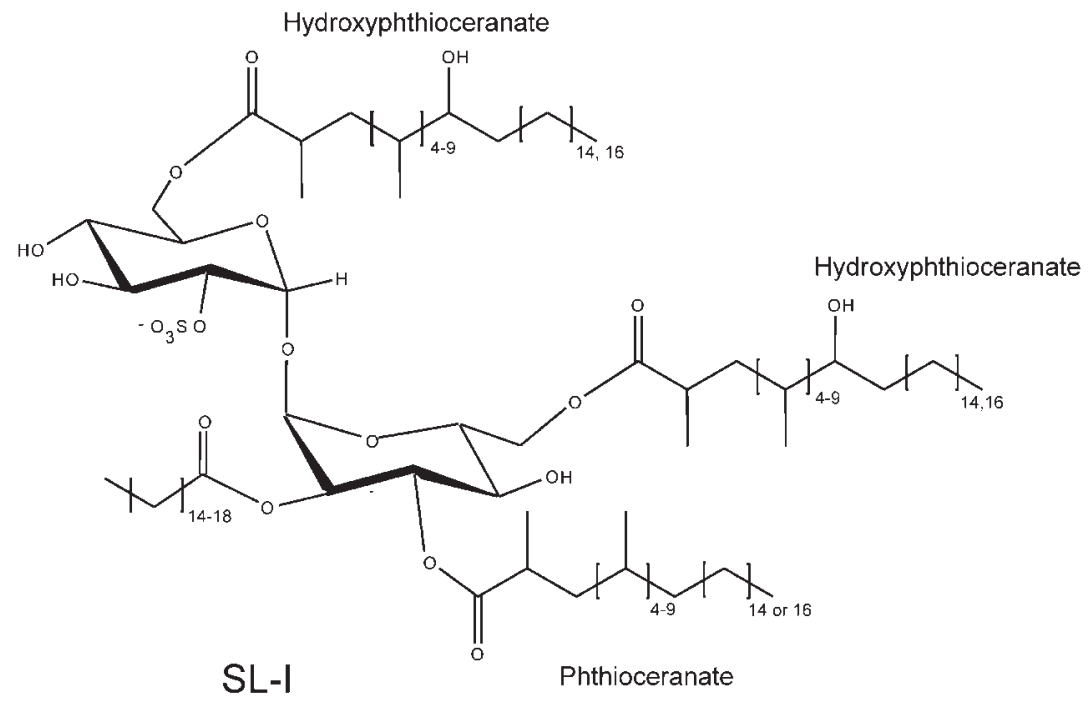

Fig. 1. Structure of sulfolipid-I (SL-I) from M. tuberculosis.

Trivedi et al., 2005). In general, pks clusters in M. tuberculosis are associated with only one PAP-encoding gene (Cole et al., 1998). Interestingly, Rv3820c (papA2), a gene located $5.8 \mathrm{~kb}$ downstream of papA1 (Fig. 2a), also encodes a protein with an acyltransferase motif, indicating that SL-I biosynthesis may involve more than one PAP. A similar arrangement of homologues of $p k s 2, p a p A 1$ and $p a p A 2$ is also seen in the genome of the bovine pathogen Mycobacterium bovis (Garnier et al., 2003), although the M. bovis-derived vaccine strain BCG does not synthesize SL-I (Rivera-Marrero et al., 2002). In these studies we have used a genetic approach to investigate whether $p a p A 1$ and $p a p A 2$ play a role in SL-I biosynthesis. Individual null mutants of $\mathrm{H} 37 \mathrm{Rv}$ papA1 and papA2 failed to produce SL-I. However, no intermediates were observed in either of the mutants, indicating an essential role for each acyltransferase in assembly of complete SL-I.

\section{METHODS}

Plasmids, phages and culture conditions. Plasmids and phages used in this study are listed in Table 1. Mycobacterium tuberculosis $\mathrm{H} 37 \mathrm{Rv}$ was cultured in $7 \mathrm{H} 9$ broth (Difco) containing $10 \%$ Middlebrook OADC enrichment and $0.05 \%$ Tween 80 , or on Middlebrook 7H10 agar (Difco); Escherichia coli strains were cultured in LB broth. The concentrations of antibiotics used were $75 \mu \mathrm{g}$ hygromycin $\mathrm{ml}^{-1}$ and $20 \mu \mathrm{g}$ kanamycin $\mathrm{ml}^{-1}$ for $\mathrm{H} 37 \mathrm{Rv}$, and $150 \mu \mathrm{g}$ hygromycin $\mathrm{ml}^{-1}$ and $40 \mu \mathrm{g}$ kanamycin $\mathrm{ml}^{-1}$ for E. coli.

Construction of null mutants. For generating an allelic-exchange construct designed to replace the papAl gene with a hygromycin resistance cassette $(h y g), \sim 1 \mathrm{~kb}$ sequences flanking the left and right of the $M$. tuberculosis $\mathrm{H} 37 \mathrm{Rv}$ papA1 gene were PCR amplified from genomic DNA using the primer pairs papA1L1 (5'-CGCACTAGTGTGTTCTCCAGCAGCAACGG-3')/papA1L2 (5' AGCAGATCTCGAGGTGTACTCGTGCTGCC- $\left.3^{\prime}\right)$ and papA1R1 (5'-TGCTCTAGAGAAAGGATGACATGGCGGTC-3')/papA1R2 (5' AGACTTAAGGCAACGAGGCGCTGTGCAAC-3') respectively. Following cloning into pCR2.1-TOPO and sequencing, the cloned PCR fragments were excised using the primer-introduced restriction sites and cloned into the allelic-exchange plasmid vector pJSC347 (Table 1). The resultant plasmid, pYUB2418, was then packaged into the temperature-sensitive phage phAE159 (J. Kriakov \& W. R. Jacobs Jr., unpublished results) as described previously (Bardarov et al., 2002) to yield the papA1-knockout phage phAE405. In a similar fashion the primer pairs papA2L1 (5'-CGGACTAGTCAGCTTATGCGCACCAC-3')/papA2L2 (5'-AATCTCGAGCGGTGATGCGTGCCAAC- $\left.3^{\prime}\right)$ and papA2R1 (5'-CGGTCTAGAGTCGCCAATTACATCGCG-3')/papA2R2 (5'-AATGGTACCAGTGGCGTCTGGACGACG-3') were used to PCR amplify $\sim 1 \mathrm{~kb}$ sequences flanking the papA2 gene. Following sequence confirmation, the flanks were used to construct the allelic-exchange plasmid pYUB2419, which was then packaged into phAE159 to yield the papA2-knockout phage phAE406. The phages were used to generate allelic-exchange mutants using specialized transduction as described previously (Bardarov et al., 2002).

Complementation of mutant strains. The primers papAl-F $\left(5^{\prime}-\right.$ CATGAATTCGTGCGAATAGGACC-3') and papA1-R (5'-GGCGTTAACCTAAGCTTCTCTATC- $3^{\prime}$ ) were used to PCR amplify the papA1 gene from $\mathrm{H} 37 \mathrm{Rv}$ genomic DNA. Following cloning into pCR2.1-TOPO and sequencing, the cloned PCR fragment was excised by digestion of the primer-introduced EcoRI and HpaI sites and cloned downstream of the $h s p 60$ promoter in the integrative vector pMV361 (Stover et al., 1991). The resultant plasmid pMV361papA1 was introduced into $\triangle p a p A 1$ by electroporation to yield the complemented strain $\triangle p a p A 1::$ pMV361papA1. Similarly, the primers papA2-F ( $5^{\prime}$-CTAAGCTTGTGTTTAGCATTACAAC- $\left.3^{\prime}\right)$ and papA2-R (5'-CGATCGATTCATGTGCCTGGTTTAAG-3') were used to PCR amplify the papA2 gene. Following subcloning and sequence confirmation, the PCR product was excised and cloned as a HindIII-ClaI fragment into pMV361 to yield the complementing plasmid pMV361papA2, which was then electroporated into $\triangle p a p A 2$ to yield the complemented strain $\triangle p a p A 2:$ pMV361papA2.

The acyltransferase motif of papAl was mutated using the Stratagene Quik Change site-directed mutagenesis kit, with pMV361papA1 as template DNA and the primer pairs papA1HA-F (5'-CAGCATCGATGCTCTGCATGCG-3')/papA1HA-R (5' -CGCATGCAGAGCATCGATGCTG-3') or papA1DA-F (5'-TCTGCATGCGGCCGGTCAGTTCG-3')/papA1DA-R (5'-CGAACTGACCGGCCGCATGCAGA-3'). The resultant plasmids, pMV361A1-HA or pMV361A1-DA, were then electroporated into $\triangle p a p A 1$. In a similar fashion, the plasmids pMV361A2-HA and pMV361A2-DA were constructed using the primer pairs papA2HA-F (5'-GAGTATCGCTGCTCTCTGTGTC- $\left.{ }^{\prime}\right)$ /papA2HA-R (5'-GACACAGAGAGCAGCGATACTC- $3^{\prime}$ ) and papA2DA-F (5'-CTCTGTGTCGCCCCGATGATTG-3')/papA2DA-R (5'-CAATCATCGGGGCGACACAGAG- $3^{\prime}$ ) respectively, and then electroporated into $\triangle p a p A 2$. 
(a)

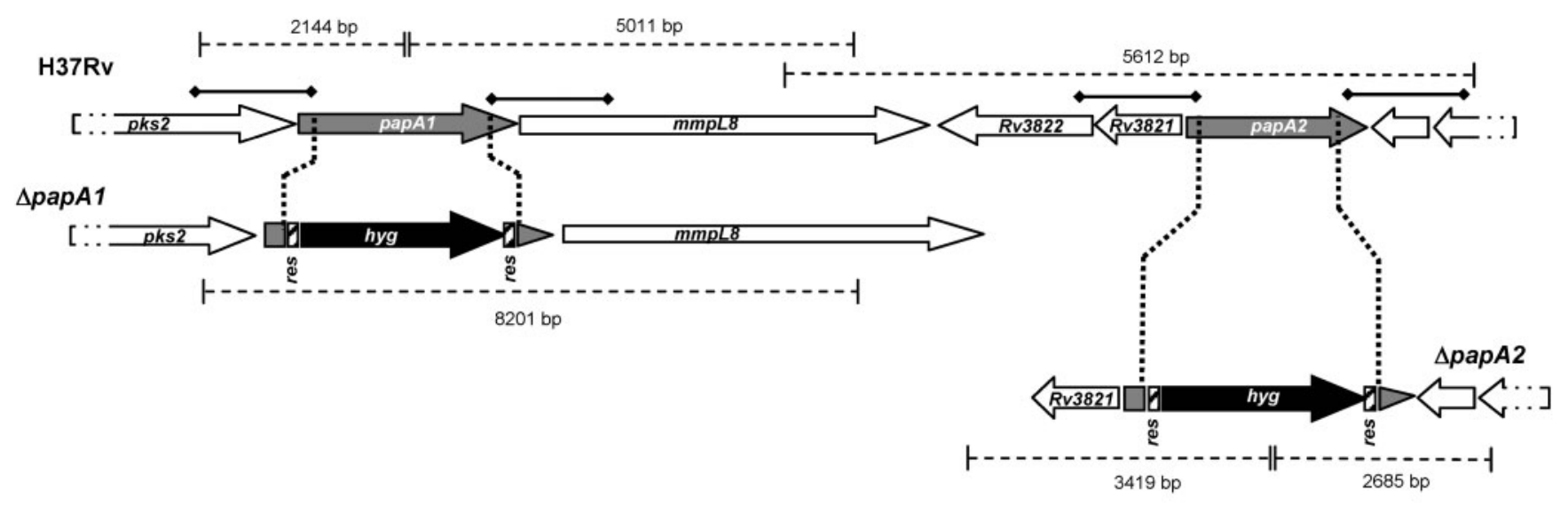

(b)

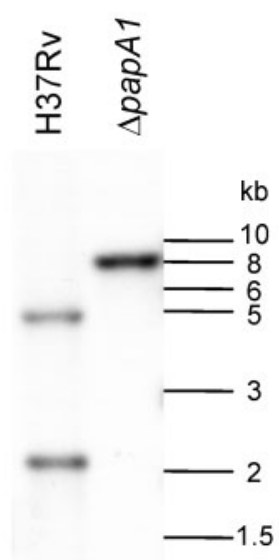

(c)

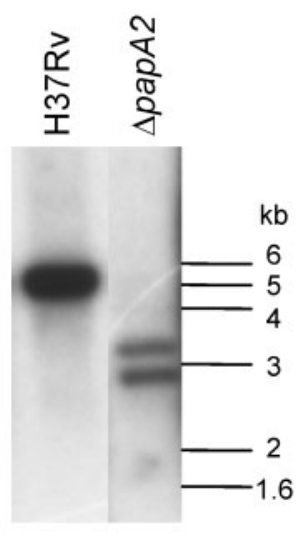

Fig. 2. Generation of $M$. tuberculosis papA1 and papA2 null mutants. (a) Maps of the $M$. tuberculosis pks2 region in wild-type (H37Rv), $\triangle$ papA1 and $\triangle$ papA2 strains: $\left[\alpha^{32} \mathrm{P}\right] \mathrm{dCTP}-$ labelled probes were derived from $\sim 1 \mathrm{~kb}$ upstream and downstream flanking sequences that were used to construct the knockout plasmids, and are indicated by thick lines with diamonds at each end. The fragments expected to hybridize with the probes in a Southern blot are indicated by gapped lines with sizes. res, $\gamma \delta$-resolvase site; hyg, hygromycin resistance gene. (b) Southern blot of Kpnl-digested genomic DNA from H37Rv and $\triangle p a p A 1$ strains. (c) Southern blot of Bbsl-digested genomic DNA from $\mathrm{H} 37 \mathrm{v}$ and $\triangle p a p A 2$ strains.
Biochemical analysis of mutant strains. Labelling of $M$. tuberculosis cultures with $\left[{ }^{14} \mathrm{C}\right]$ acetate, $\left[{ }^{14} \mathrm{C}\right]$ propionate or $\mathrm{Na}_{2}{ }^{35} \mathrm{SO}_{4}$ was done as described previously (Sirakova et al., 2001). Total lipids were extracted from strains using previously described protocols (Dobson et al., 1985) and separated on a TLC plate using chloroform/metha$\mathrm{nol} /$ water $(100: 14: 0.8$, by vol.) in the first dimension and chloroform/acetone/methanol/water $(50: 60: 2.5: 3$, by vol.) in the second dimension. The TLC plates were exposed to X-ray films for $24 \mathrm{~h}$.

\section{RESULTS}

\section{Generation of papA1 and papA2 null mutants of M. tuberculosis}

We used specialized transduction (Bardarov et al., 2002) to replace papA1 in the $M$. tuberculosis $\mathrm{H} 37 \mathrm{Rv}$ genome with a hygromycin resistance marker (hyg) (Fig. 2a). Genomic DNA obtained from $\mathrm{Hyg}^{\mathrm{R}}$ M. tuberculosis $\mathrm{H} 37 \mathrm{Rv}$ colonies following transduction with phAE405 was digested with KpnI and analysed by Southern blotting (Fig. 2b). The band pattern obtained confirmed that papAl was replaced by hyg. Similarly, the knockout phage phAE406 was used to obtain a papA2 null mutant of H37Rv (Fig. 2c). The papA1 and $p a p A 2$ null mutants are referred to as $\triangle p a p A 1$ and $\triangle p a p A 2$ respectively.

\section{SL-I is not synthesized in $\Delta$ papA1}

To determine changes in the lipid profiles of the $\triangle p a p A 1$ mutant, we first monitored differences in the $\left[{ }^{14} \mathrm{C}\right]$ acetatelabelled lipids from wild-type and $\triangle p a p A 1$ strains by TLC. In a solvent system designed to separate $M$. tuberculosis glycolipids (Dobson et al., 1985), a ${ }^{14}$ C-labelled lipid, which was clearly present in the wild-type but missing in the mutant strain, migrated in a manner similar to an authentic SL-I standard (Fig. 3a). Three of the four acyl components of SL-I are methyl-branched fatty acids (phthioceranate and hydroxyphthioceranate) which can be labelled using $\left[{ }^{14} \mathrm{C}\right]$ propionate. Indeed, the lipid missing in $\triangle p a p A 1$, but present in WT showed high incorporation of ${ }^{14} \mathrm{C}$ label when propionate was used as the radiolabel (Fig. 3b). Finally, this lipid also showed incorporation of ${ }^{35} \mathrm{~S}$ from $\mathrm{Na}_{2}{ }^{35} \mathrm{SO}_{4}$, confirming that the species was indeed SL-I (Fig. 3b). Introduction of pMV361papA1, a single copy integrative vector containing papA1 cloned downstream of 
Table 1. Plasmids and phages

\begin{tabular}{|c|c|c|}
\hline Plasmid or phage & Description & Reference/source \\
\hline \multicolumn{3}{|l|}{ Plasmids } \\
\hline pCR2.1-TOPO & PCR product cloning vector & Invitrogen Life Technologies \\
\hline pJSC347 & $\begin{array}{l}\text { Vector for cloning allelic-exchange substrates to be used for specialized } \\
\text { transduction; contains } \lambda \text { phage } \cos \text { site and } \operatorname{Hyg}^{\mathrm{R}} \text { marker }\end{array}$ & Sambandamurthy et al. (2002) \\
\hline pYUB2418 & Derivative of pJSC347 designed for allelic exchange of $M$. tuberculosis papA1 & This work \\
\hline pYUB2419 & Derivative of pJSC347 designed for allelic exchange of $M$. tuberculosis papA2 & This work \\
\hline pMV361 & Single-copy mycobacterial integrative vector with $h s p 60$ promoter & Stover et al. (1991) \\
\hline pMV361papA1 & M. tuberculosis papA1 cloned in pMV361 & This work \\
\hline pMV361A1-HA & His-171 $\rightarrow$ Ala-171 mutant of papA1 in pMV361 & This work \\
\hline pMV361A1-DA & Asp-175 $\rightarrow$ Ala-175 mutant of papA1 in pMV361 & This work \\
\hline pMV361 papA2 & M. tuberculosis papA2 cloned in pMV361 & This work \\
\hline pMV361A2-HA & His- $166 \rightarrow$ Ala-166 mutant of papA2 in pMV361 & This work \\
\hline pMV361A2-DA & Asp-170 $\rightarrow$ Ala-170 mutant of papA2 in pMV361 & This work \\
\hline \multicolumn{3}{|l|}{ Phages } \\
\hline phAE159 & $\begin{array}{l}\text { Conditionally replicating shuttle phasmid derived from the lytic } \\
\text { mycobacteriophage TM4 }\end{array}$ & Gift from J. Kriakov \\
\hline phAE405 & Derivative of phAE159 obtained by cloning pYUB2418 in its unique PacI site & This work \\
\hline phAE406 & Derivative of phAE159 obtained by cloning pYUB2419 in its unique PacI site & This work \\
\hline
\end{tabular}

${ }^{*}$ Albert Einstein College of Medicine.

the hsp60 promoter, into $\triangle p a p A 1$ fully restored SL-I biosynthesis, indicating that the observed phenotype in $\triangle p a p A 1$ was solely due to deletion of papA1 (Fig. 3a, b). These results demonstrated that papA1 function was necessary for SL-I biosynthesis.

\section{PapA2 is also required for SL-I biosynthesis}

To investigate the role of papA2 in SL-I biosynthesis, an approach similar to that used for the $\triangle p a p A 1$ strain was followed. An initial analysis of total lipids extracted from cultures of H37Rv and $\triangle p a p A 2$ labelled with $\left[{ }^{14} \mathrm{C}\right]$ acetate indicated that SL-I was missing in the $\triangle p a p A 2$ strain (Fig. 4a). This was then confirmed using $\left[{ }^{14} \mathrm{C}\right]$ propionate or $\mathrm{Na}_{2}{ }^{35} \mathrm{SO}_{4}$ as the labelling reagents (Fig. 4b). SL-I biosynthesis could be restored in $\triangle p a p A 2$ following complementation with pMV361papA2, a single-copy integrative vector containing $p a p A 2$ cloned downstream of the $h s p 60$ promoter (Fig. 4a, b). Thus, like papA1, papA2 was also essential for SL-I biosynthesis.

\section{papA1 and papA2 encode acyltransferases}

The presence of a conserved acyltransferase motif $\left(\mathrm{HX}_{3} \mathrm{DX}_{14} \mathrm{Y}\right)$ had suggested a role for PapA1 and PapA2 in acylation of either trehalose or sulfated trehalose, to form SL-I. The histidine and aspartate residues of the motif are considered essential for acyltransferase activity (Bergendahl et al., 2002; Lewendon et al., 1988). To demonstrate that papA 1 and $p a p A 2$ do encode acyltransferases, we tested the ability of mutant alleles of the genes containing alterations in either of these key residues to restore SL-I biosynthesis in the mutant strain. First, single-copy-integrative plasmids containing two mutant alleles of papA1 containing separate point mutations in the $\mathrm{HX}_{3} \mathrm{DX}_{14} \mathrm{Y}$ motif were constructed using site-directed mutagenesis (Fig. 5). The mutant constructs pMV361A1-HA (His-171 $\rightarrow$ Ala-171) and pMV361A1-DA (Asp-175 $\rightarrow$ Ala-175) were then introduced separately into $\triangle p a p A 1$ by electroporation and the resultant strains, $\triangle p a p A 1:: \mathrm{pMV} 361 A 1-H A$ and $\triangle p a p A 1::$ pMV361A1-DA, were tested for functional complementation. Analysis of $\left[{ }^{14} \mathrm{C}\right]$ propionate- or ${ }^{35} \mathrm{~S}$-labelled total lipids from the transformed strains revealed that neither construct reconstituted SL-I biosynthesis in $\triangle p a p A 1$ (Fig. 3b). Similarly, the plasmids pMV361A2-HA (His$166 \rightarrow$ Ala-166) and pMV361A2-DA (Asp-170 $\rightarrow$ Ala-170) failed to restore SL-I biosynthesis in $\triangle p a p A 2$ (Fig. 4b). Thus, the key residues in the putative acyltransferase motifs of PapA1 and PapA2 were essential for functional complementation of the corresponding SL-I deficient mutants, indicating that PapA1 and PapA2 are acyltransferases involved in SL-I biosynthesis.

\section{DISCUSSION}

The sequencing of the M. tuberculosis H37Rv genome revealed five open reading frames, $p a p A 1-p a p A 5$, that were annotated as genes for polyketide synthase-associated proteins (PAPs) because of their linkage to genes encoding enzymes involved in polyketide biosynthesis (Cole et al., 1998). Based on the presence of an acyltransferase motif, it was speculated that PAPs may be acyltransferases involved in the assembly of complex lipid metabolites synthesized by the associated pks cluster. Experimental proof for this came first from in vitro experiments using purified PapA5, a PAP 
(a)
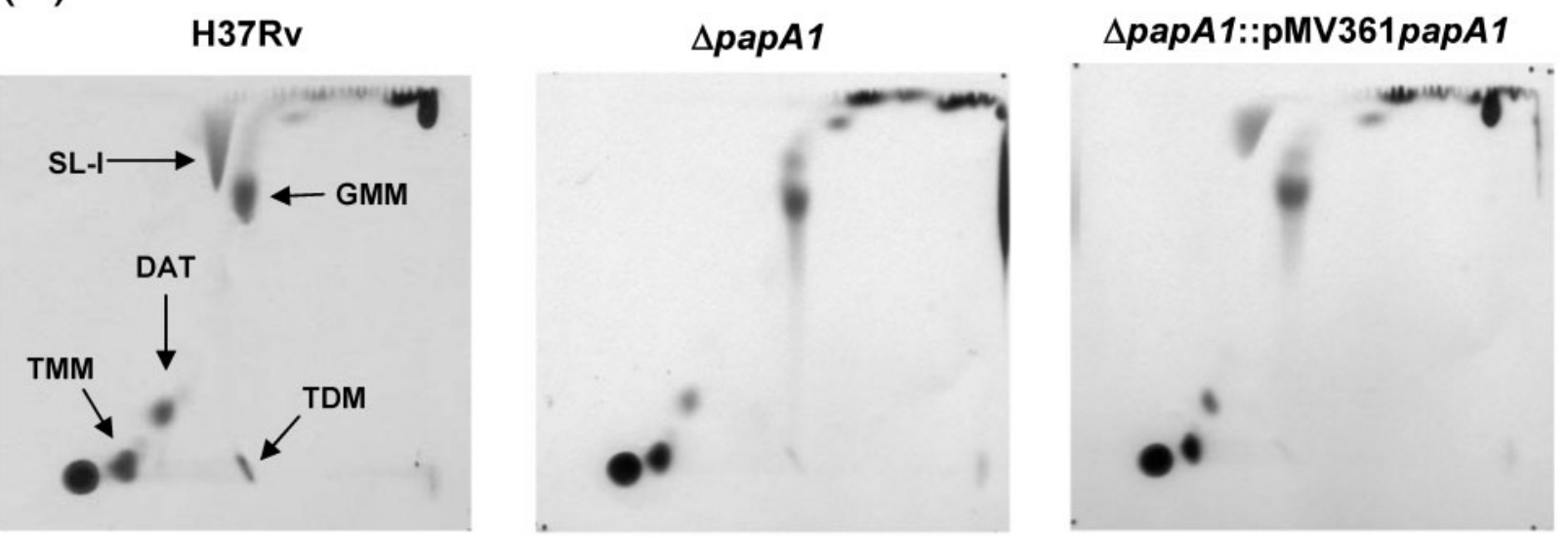

(b)
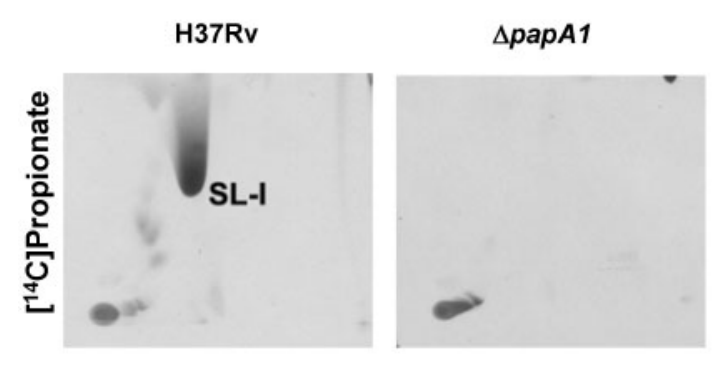

АpapA1::pMV361papA1

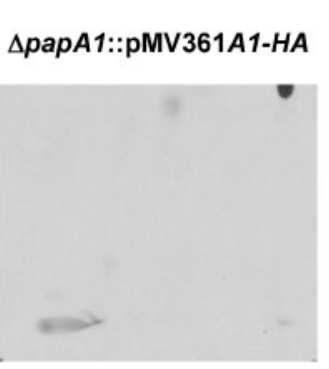

ApapA1::pMV361A1-DA
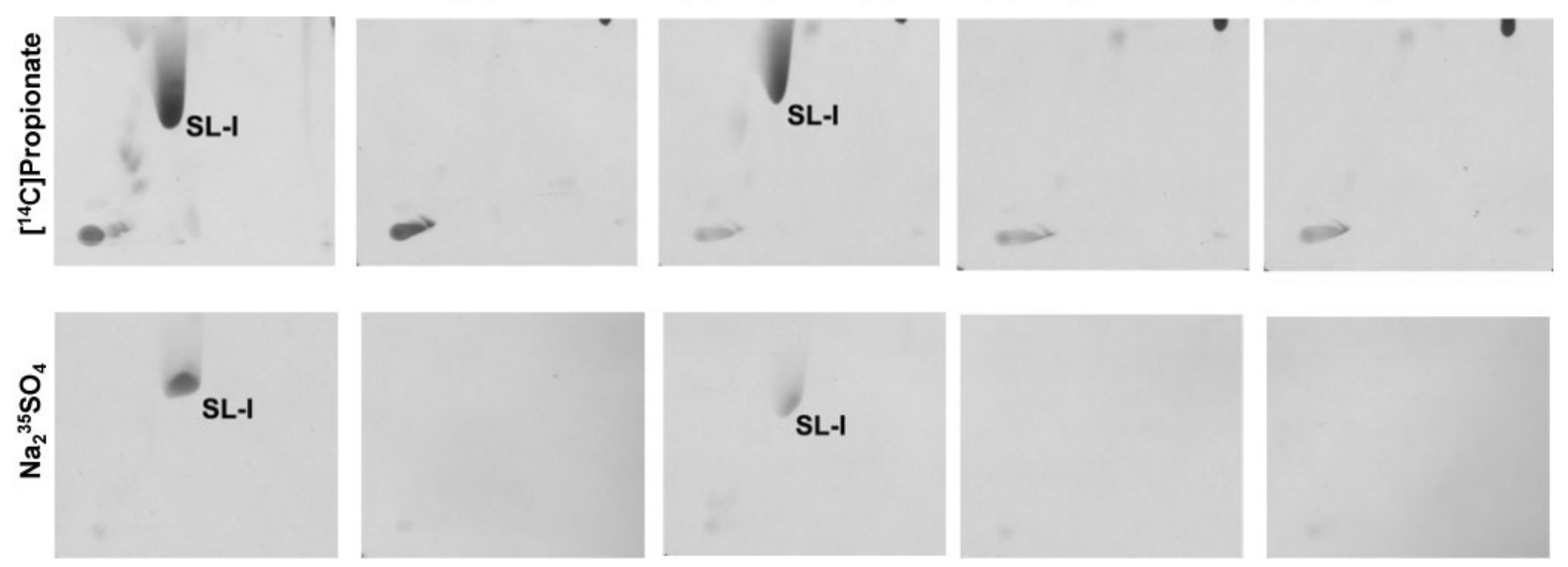

Fig. 3. Sulfolipid analysis of the $\triangle p a p A 1$ mutant by 2D-TLC. X-ray film exposed to TLC plates following $2 \mathrm{D}-\mathrm{TLC}$ of total lipid samples extracted from cultures labelled with (a) $\left[{ }^{14} \mathrm{C}\right]$ acetate or (b) $\left[{ }^{14} \mathrm{C}\right]$ propionate or $\mathrm{Na}_{2}{ }^{35} \mathrm{SO}_{4}$. The spot corresponding to SL-I is indicated in the images. GMM, glucose monomycolate; DAT, diacyltrehalose; TMM, trehalose monomycolate; TDM, trehalose dimycolate.

involved in PDIM biosynthesis (Onwueme et al., 2004; Trivedi et al., 2005). PDIM is made up of two mycocerosic acid residues esterified to the hydroxyl groups of a diol (phthiocerol). Using purified proteins and artificial substrates, Onwueme et al. (2004) first showed that PapA5 could catalyse esterification of an alcohol with an acyl group. Trivedi et al. (2005) then demonstrated that purified PapA5 catalysed trans-esterification of mycocerosic acid synthasebound mycocerosic acids to dodecanol.

Of all the pks genes linked to PAPs, pks2 is the only one located next to two pap genes ( $p a p A 3$ and $p k s 3 / 4$ are involved in the biosynthesis of polyacylated trehalose, and the metabolite synthesized by papA4 and pks5 has not yet been identified). Deletion of $p k s 2$ resulted in loss of the principal sulfolipid SL-I in M. tuberculosis H37Rv (Sirakova et al., 2001). The absence of SL-I in $\triangle p a p A 1$ and in $\triangle p a p A 2$, the two mutants generated in this study, demonstrated that PapA1 and PapA2 were indeed functionally associated with Pks2. Phthioceranic acid and hydroxyphthioceranic acid, the methyl-branched products of Pks2, are found esterified to positions 3 , and 6 and $6^{\prime}$, respectively, of sulfated trehalose in the principal sulfolipid SL-I, while position 2 is substituted with FAS-I-derived palmitate. It was thus tempting to speculate that PapA1 and PapA2 may differ in their substrate specificities, with one PAP involved in the transfer of the methyl-branched acyl chains, and the other in that of palmitate. Another possibility was that one of the pks2-associated PAPs was specific for transfer of pthioceranic acid and the other for hydroxyphthioceranic acid, with 
(a)
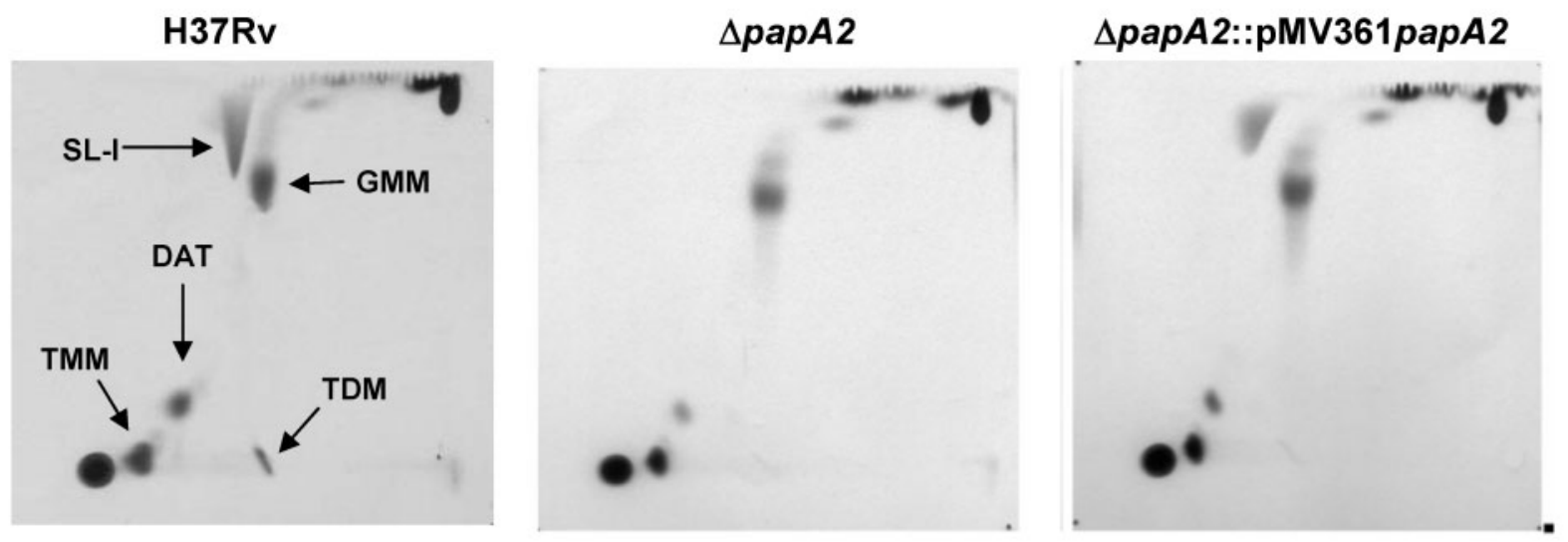

(b)
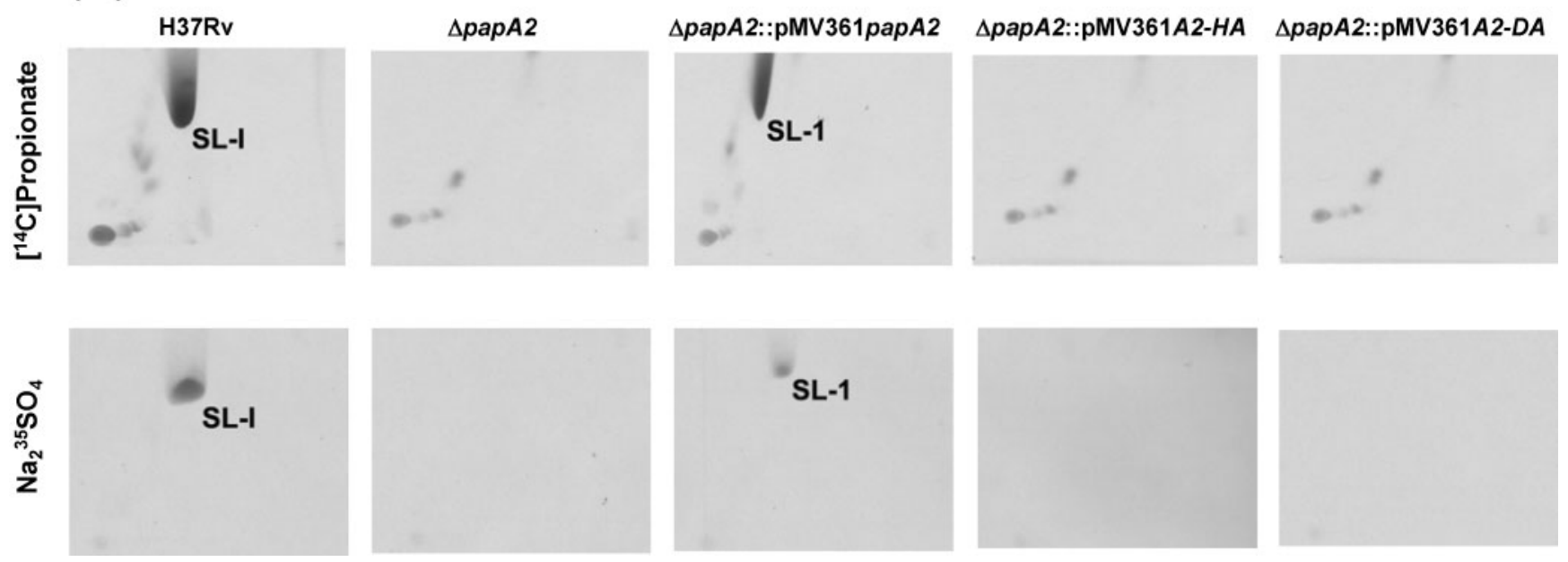

Fig. 4. Sulfolipid analysis of the $\triangle p a p A 2$ mutant by 2D-TLC. X-ray film exposed to TLC plates following $2 \mathrm{D}-\mathrm{TLC}$ of total lipid samples extracted from cultures labelled with (a) $\left[{ }^{14} \mathrm{C}\right]$ acetate or (b) $\left[{ }^{14} \mathrm{C}\right]$ propionate or $\mathrm{Na}_{2}{ }^{35} \mathrm{SO}_{4}$. The spot corresponding to SL-I is indicated in the images. GMM, glucose monomycolate; DAT, diacyltrehalose; TMM, trehalose monomycolate; TDM, trehalose dimycolate.

the transfer of palmitate being carried out by an unknown acyltransferase. In either case, if the acylation reactions were sequential, partially acylated trehalose sulfate intermediates would be expected to accumulate in either $\triangle p a p A 1$ or $\triangle p a p A 2$. While both $\triangle p a p A 1$ and $\triangle p a p A 2$ did not synthesize SL-I, neither mutant strain showed accumulation of any partially acylated intermediate. This result indicated that in one of the mutants, feedback inhibition of the initial step may have prevented the accumulation of the intermediate. Alternatively, instead of a sequential addition of different acyl groups to sulfated trehalose by PapA1 and PapA2, concurrent presence of both proteins may be required for complete assembly of a tetracylated trehalose sulfate molecule. Some indication that this might be a possibility comes from recent work on the components of the PDIM biosynthetic cluster. Jain \& $\operatorname{Cox}$ (2005) showed that polyketide synthase PpsE directly interacted with the transmembrane protein and PDIM transporter MmpL7. This suggested that not only was MmpL7 involved in transport, but that it also probably acted as a scaffold in a tightly integrated system that couples the biosynthesis of PDIM to its secretion from the bacterial cell. In light of these findings it is plausible that MmpL8, the transporter associated with the pks2 cluster, may play a similar 'scaffolding' role in SL-I assembly through interactions with Pks2, PapA1 and Pap2, wherein PapA1 and PapA2 may carry out localized and simultaneous acylation reactions. In the $\triangle p a p A 1$ or $\triangle p a p A 2$ mutant, loss of one PAP would result in non-functional SL-I assembly machinery and complete loss of SL-I biosynthesis (rather than accumulation of a partially acylated intermediate). However, two independent reports of a $M$. tuberculosis mmpL8 null mutant showed that 


\section{PapA1 \\ A $1-H A$ \\ A1-DA \\ PapA2 \\ A2-HA \\ $\mathrm{A} 2-\mathrm{DA}$ \\ Motif consensus HXXXDXXXXXXXXXXXXXXY}

Fig. 5. Mutation of the $H_{X_{3}} D X_{14} Y$ motif in PapA1 and PapA2. Alignment of the amino acid sequences in the $\mathrm{HX}_{3} \mathrm{DX} \mathrm{1}_{14} \mathrm{Y}$ motif of PapA1, PapA2 and the putative proteins encoded by the mutant constructs pMV361A1-HA (A1-HA), pMV361A1-DA (A1-DA), pMV361A2-HA (A2-HA), pMV361A2-DA (A2-DA). The conserved amino acids are indicated in bold and the altered residues are underlined.

the loss of MmpL8 resulted in the accumulation of a diacylated trehalose sulfate intermediate (Converse et al., 2003; Domenech et al., 2004). Nevertheless, we did not detect accumulation of such an intermediate in either the $\triangle p a p A 1$ or the $\triangle p a p A 2$ mutant. Though the absence of any partially acylated intermediates in either $\triangle p a p A 1$ or $\triangle p a p A 2$ made it difficult to assign putative substrate specificities (if any) to PapA1 and PapA2, our results have clearly demonstrated that these two distinct acyltransferases are involved in the biosynthesis of the major sulfolipid SL-I in M. tuberculosis.

\section{ACKNOWLEDGEMENTS}

The authors would like to thank Nagatoshi Fujiwara for helpful suggestions on sulfolipid analysis and Catherine Vilchèze for comments on the manuscript. This work was supported by grants from NIH AI26170 and CFAR (AI 051519) to W.R. J.; G.S.B. acknowledges support from Mr James Bardrick in the form of a Personal Research Chair, and as a former Lister Institute Jenner Research Fellow, and of the Medical Research Council (UK).

\section{REFERENCES}

Bardarov, S., Bardarov, S., Jr, Pavelka, M. S., Jr, Sambandamurthy, V., Larsen, M., Tufariello, J., Chan, J., Hatfull, G. \& Jacobs, W. R., Jr (2002). Specialized transduction: an efficient method for generating marked and unmarked targeted gene disruptions in Mycobacterium tuberculosis, M. bovis BCG and M. smegmatis. Microbiology 148, 3007-3017.

Bergendahl, V., Linne, U. \& Marahiel, M. A. (2002). Mutational analysis of the C-domain in nonribosomal peptide synthesis. Eur J Biochem 269, 620-629.

Brennan, P. J. \& Nikaido, H. (1995). The envelope of mycobacteria. Annu Rev Biochem 64, 29-63.

Cole, S. T., Brosch, R., Parkhill, J. Garnier T., Churcher, C. Harris D., Gordon, S. V., Eiglmeier, K., Gas, S. \& other authors (1998). Deciphering the biology of Mycobacterium tuberculosis from the complete genome sequence. Nature 393, 537-544.
Converse, S. E., Mougous, J. D., Leavell, M. D., Leary, J. A., Bertozzi, C. R. \& Cox, J. S. (2003). MmpL8 is required for sulfolipid-1 biosynthesis and Mycobacterium tuberculosis virulence. Proc Natl Acad Sci U S A 100, 6121-6126.

Dobson, G., Minnikin, D. E., Minnikin, S. M., Parlett, M., Goodfellow, M., Ridell, M. \& Magnusson, M. (1985). Systematic analysis of complex mycobacterial lipids. In Chemical Methods in Bacterial Systematics, pp. 237-265. Edited by M. Goodfellow \& D. E. Minnikin. London: Academic Press.

Domenech, P., Reed, M. B., Dowd, C. S., Manca, C., Kaplan, G. \& Barry, C. E., 3rd (2004). The role of MmpL8 in sulfatide biogenesis and virulence of Mycobacterium tuberculosis. J Biol Chem 279, 21257-21265.

Garnier, T., Eiglmeier, K., Camus, J. C., Medina, N., Mansoor, H., Prior, M., Duthoy, S., Grondin, S., Lacroix, C. \& other authors (2003). The complete genome sequence of Mycobacterium bovis. Proc Natl Acad Sci U S A 100, 7877-7882.

Goren, M. B. (1970a). Sulfolipid I of Mycobacterium tuberculosis, strain H37Rv. II. Structural studies. Biochim Biophys Acta 210, 127-138.

Goren, M. B. (1970b). Sulfolipid I of Mycobacterium tuberculosis, strain H37Rv. I. Purification and properties. Biochim Biophys Acta 210, 116-126.

Goren, M. B., Brokl, O., Das, B. C. \& Lederer, E. (1971). Sulfolipid I of Mycobacterium tuberculosis, strain H37RV. Nature of the acyl substituents. Biochemistry 10, 72-81.

Goren, M. B., Brokl, O. \& Das, B. C. (1976). Sulfatides of Mycobacterium tuberculosis: the structure of the principal sulfatide (SL-I). Biochemistry 15, 2728-2735.

Jain, M. \& Cox, J. S. (2005). Interaction between polyketide synthase and transporter suggests coupled synthesis and export of virulence lipid in M. tuberculosis. PLoS Pathog 1, e2.

Lewendon, A., Murray, I. A., Kleanthous, C., Cullis, P. M. \& Shaw, W. V. (1988). Substitutions in the active site of chloramphenicol acetyltransferase: role of a conserved aspartate. Biochemistry 27, 7385-7390.

Middlebrook, G., Coleman, C. M. \& Schaefer, W. B. (1959). Sulfolipid from virulent tubercle bacilli. Proc Natl Acad Sci U S A 45, 1801-1804.

Mougous, J. D., Petzold, C. J., Senaratne, R. H., Lee, D. H., Akey, D. L., Lin, F. L., Munchel, S. E., Pratt, M. R., Riley, L. W. \& other authors (2004). Identification, function and structure of the mycobacterial sulfotransferase that initiates sulfolipid-1 biosynthesis. Nat Struct Mol Biol 11, 721-729.

Okamoto, Y., Fujita, Y., Naka, T., Hirai, M., Tomiyasu, I. \& Yano, I. (2006). Mycobacterial sulfolipid shows a virulence by inhibiting cord factor induced granuloma formation and TNF-alpha release. Microb Pathog 40, 245-253.

Onwueme, K. C., Ferreras, J. A., Buglino, J., Lima, C. D. \& Quadri, L. E. (2004). Mycobacterial polyketide-associated proteins are acyltransferases: proof of principle with Mycobacterium tuberculosis PapA5. Proc Natl Acad Sci U S A 101, 4608-4613.

Pabst, M. J., Gross, J. M., Brozna, J. P. \& Goren, M. B. (1988). Inhibition of macrophage priming by sulfatide from Mycobacterium tuberculosis. J Immunol 140, 634-640.

Puzo, G. (1990). The carbohydrate- and lipid-containing cell wall of mycobacteria, phenolic glycolipids: structure and immunological properties. Crit Rev Microbiol 17, 305-327.

Rivera-Marrero, C. A., Ritzenthaler, J. D., Newburn, S. A., Roman, J. \& Cummings, R. D. (2002). Molecular cloning and expression of a novel glycolipid sulfotransferase in Mycobacterium tuberculosis. Microbiology 148, 783-792. 
Rousseau, C., Turner, O. C., Rush, E., Bordat, Y., Sirakova, T. D., Kolattukudy, P. E., Ritter, S., Orme, I. M., Giquel, B. \& Jackson, M. (2003). Sulfolipid deficiency does not affect the virulence of Mycobacterium tuberculosis $\mathrm{H} 37 \mathrm{Rv}$ in mice and guinea pigs. Infect Immun 71, 4684-4690.

Sambandamurthy, V. K., Wang, X., Chen, B., Russell, R. G., Derrick, S., Collins, F. M., Morris, S. L. \& Jacobs, W. R., Jr (2002). A pantothenate auxotroph of Mycobacterium tuberculosis is highly attenuated and protects mice against tuberculosis. Nat Med 8, 1171-1174.

Sirakova, T. D., Thirumala, A. K., Dubey, V. S., Sprecher, H. \& Kolattukudy, P. E. (2001). The Mycobacterium tuberculosis pks2 gene encodes the synthase for the hepta- and octamethyl-branched fatty acids required for sulfolipid synthesis. J Biol Chem 276, 16833-16839.
Stover, C. K., de la Cruz, V. F., Fuerst, T. R., Burlein, J. E., Benson, L. A., Bennett, L. T., Bansal, G. P., Young, J. F., Lee, M. H. \& other authors (1991). New use of BCG for recombinant vaccines. Nature 351, 456-460.

Trivedi, O. A., Arora, P., Vats, A., Ansari, M. Z., Tickoo, R., Sridharan, V., Mohanty, D. \& Gokhale, R. S. (2005). Dissecting the mechanism and assembly of a complex virulence mycobacterial lipid. Mol Cell 17, 631-643.

Zhang, L., Goren, M. B., Holzer, T. J. \& Andersen, B. R. (1988). Effect of Mycobacterium tuberculosis-derived sulfolipid I on human phagocytic cells. Infect Immun 56, 2876-2883.

Edited by: S. V. Gordon 